\title{
Merkel Cell Carcinoma of the Hand: A Case Report and Review of the Literature
}

HAND

2016, Vol. II (4) NP24-NP29

(C) American Association for

Hand Surgery 2016

DOI: $10.1177 / 15589447|56| 6098$

hand.sagepub.com

\author{
Donevan R. Westerveld', David J. Hall', and Winston T. Richards'
}

\begin{abstract}
Background: Merkel cell carcinoma (MCC) is a relatively rare and aggressive cutaneous neuroendocrine malignancy characterized by high incidence of local recurrence, distant metastases, regional nodal metastases, and high mortality. Clinically, MCC presents as a persistent asymptomatic red/pink shaped nodule, usually smaller than $2 \mathrm{~cm}$ with nontender intracutaneous swelling, with rapidly growing localized disease with lymph node metastases preceding distant metastases. Because of its rare nature and the lack of comprehensive understanding of the disease, management of MCC has been controversial. Methods: An 87-year-old retired Caucasian male with a history of tobacco use, chronic sun exposure, and multiple squamous and basal cell carcinomas presented with a $1.8 \times 1.3 \mathrm{~cm}$ red, nontender nodule on the dorsum of the proximal phalanx of the left long finger first noticed 6 months prior to presentation. Biopsy was consistent with MCC after which he was treated with wide local excision, full-thickness skin grafting, and sentinel lymphadenectomy (I/4 nodes positive) followed by adjuvant radiation therapy. Results: He recovered appropriately and was clinically and radiographically disease free at 2.5-year follow-up. Conclusion: Although it remains rare, MCC has increased in incidence over the last several decades and has a predilection to occur over sun exposed areas. Highly aggressive, it has a high incidence of regional and distant metastasis as well as local recurrence. As a result, it is important that practitioners involved in the care of skin and hand lesions be aware of this condition and the need for a multidisciplinary treatment approach.
\end{abstract}

Keywords: Merkel cell carcinoma, hand, MCC, neurocutaneous, radiation

\section{Introduction}

Merkel cell carcinoma (MCC) is a relatively rare and aggressive cutaneous neuroendocrine malignancy first described by Toker in $1972 .{ }^{28}$ An inherently aggressive disease, MCC is biologically characterized by high incidence of local recurrence, distant metastases, regional nodal metastases, and high mortality. ${ }^{1,17}$ These factors in combination with often delayed presentation make MCC a challenge to control. It is believed to arise from the Merkel's cell, first described by Friedrich Sigmund Merkel, a histopathologist, in 1875 as Tastzellen (touch cells). These neurotactile cells of neuroendocrine origin are found in the stratum basale of the epidermis with a high density of these cells located on hairless skin such as the lips, fingers, palms, and soles of the feet, and function as mechanoreceptors of light touch discrimination of shapes and textures..$^{20}$ Arising in the dermis, MCC extends down into the subcutis; however, because epidermis is rarely involved, the presentation of ulcerated skin is rare.

MCC presents on the extremities (in 38\% of cases) and head and neck $(29 \%),{ }^{3}$ suggesting a correlation with ultraviolet $\mathrm{B}$ radiation or sun exposure. There are several other risk factors associated with MCC: immunosuppression, history of organ transplantation, and Merkel cell polyomavirus infection. ${ }^{1,9,15}$ Furthermore, there appears to be an advanced age predilection as the mean age for initial diagnosis for men is 73 years and 76 years for women. ${ }^{2}$

Clinically, MCC presents as a persistent asymptomatic red/pink shaped nodule, and less frequently as blue/violaceous. Typically, they are smaller than $2 \mathrm{~cm}$ with nontender intracutaneous swelling. ${ }^{3,12,23}$ In addition, most cases present as rapidly growing localized disease with lymph node metastases preceding distant metastases. ${ }^{21}$ Because of its rare nature and the lack of comprehensive understanding of the disease, the management of MCC has been controversial. Surgical excision and sentinel lymph node biopsy

'University of Florida College of Medicine, Gainesville, FL, USA

Corresponding Author:

Winston T. Richards, Clinical Assistant Professor, Department of Surgery, University of Florida College of Medicine, 1600 SW Archer Road, Box 100286, Gainesville, FL 32610, USA.

Email: winston.richards@surgery.ufl.edu 


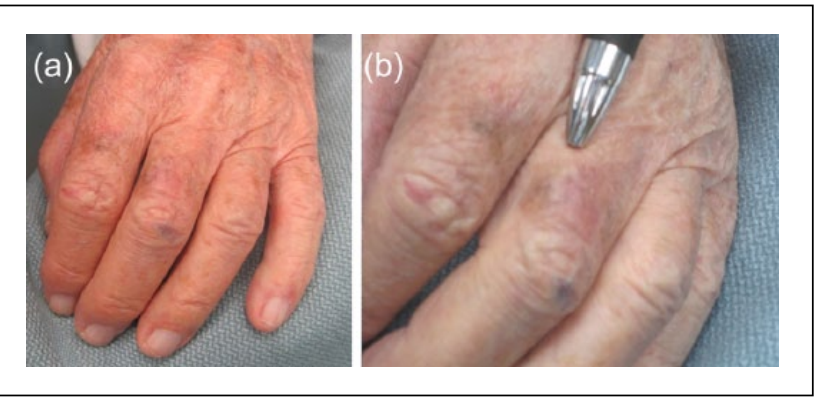

Figure I. Initial clinic presentation at (a) low magnification and (b) higher magnification review emphasizes the slightly raised and discolored area on the left long finger over the proximal phalanx.

(SLNB) followed by site-specific irradiation remains the standard of care. Five clinical features most commonly presented with MCC are represented by the acronym, AEIOU, or Asymptomatic/lack of tenderness, Expanding rapidly ( $\leq 3$ months), Immunosuppression, Older than 50 years of age, anatomical location on an ultraviolet (UV) exposed site. ${ }^{12}$

An estimated age-adjusted annual incidence of 0.32 per 100000 people per year, but more importantly, an increase in MCC incidence from 0.15 cases per 100000 in 1986 to 0.44 cases in $2001,{ }^{1,13}$ highlights the importance of controlling this very rare yet potentially fatal cancer.

\section{Case Presentation}

An 87-year-old retired Caucasian male with a history of tobacco use, chronic sun exposure, and multiple squamous and basal cell carcinomas, presented to the Surgical Oncology service with a small red, nontender nodule on the dorsum of the proximal phalanx of the left long finger. The patient first noticed a "bug bite" 6 months before presentation that progressively enlarged but never became painful. Local examination showed an ill-defined $1.8 \times 1.3$ $\mathrm{cm}$ area of slight roughening and tan discoloration (see Figure 1).

Biopsy was significant for MCC and the patient underwent a wide local excision (WLE) of the lesion (5.4 $\times 4.7 \times 0.2 \mathrm{~cm}$ ) with full-thickness skin graft $(\mathrm{FTSG})$ closure. Intraoperatively, sentinel lymphadenectomy of the left deep axilla was performed. Microscopic examination of the primary lesion revealed the presence of a malignant neoplasm within the dermis. Histologically, it was poorly circumscribed and nonencapsulated, formed of trabeculae of small blue cells with abundant nuclear molding, individual cell necrosis, geographic necrosis, marked pleomorphism and atypia, and numerous mitotic figures. In addition, these neoplastic cells stain positively with antibodies against both cytokeratin 20 (CK20) and

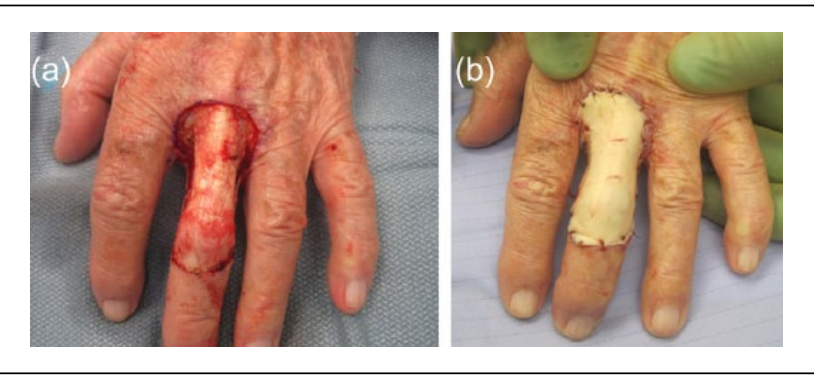

Figure 2. Intraoperative photographs demonstrating (a) fullthickness excision of the lesion with a wide $\mathrm{I}$ to $2 \mathrm{~cm}$ margin and (b) full-thickness skin graft closure of the wound.

synaptophysin, showing a characteristic paranuclear dot pattern, indicating that this was most likely a primary tumor. ${ }^{6,16}$ The excisional margins of the skin and subcutis were free of tumor. One of 4 SLNs had evidence of metastatic disease on both routine and synaptophysin staining. This metastasis was localized largely within the subcapsular sinus, focally extending into the pericapsular fatty tissue (see Figure 2).

Two months following diagnosis and excision, radiotherapy at both the primary site and wide areas of the left axilla commenced at a dose of 200 and $180 \mathrm{cGy} /$ fraction, respectively. In addition, our patient received a left axillary boost dose of $600 \mathrm{cGy}$ for a total dose of $4500 \mathrm{cGy}$ at the secondary site and $4400 \mathrm{cGy}$ at the primary site. Care and consideration of the graft was taken into account, and radiotherapy of the dorsum of the finger at the primary site was withheld for 1 week until there was a large degree of healing. Completion of nearly 1 month of radiotherapy resulted in no unusual reactions or changes in graft adherence. In clinical follow-up examinations, the left middle finger was completely healed with normal mobility and function. A positron emission tomography (PET) scan performed 8 months following excision showed no evidence of disease. Furthermore, 2-year clinical follow-up suggested no recurrent disease at the primary site (see Figure 3).

\section{Discussion}

Since initial reporting in 1972, MCC also referred to as neuroendocrine carcinoma, trabecular carcinoma, primary small cell carcinoma of the skin, and anaplastic carcinoma of the skin has been characterized as an uncommon highly aggressive malignancy that arises from the Merkel's cells located in the basal layer of the epidermis. ${ }^{20}$ This disease appears to have an age-related course of incidence with the vast majority of patients ( $72 \%$ ) over the age of 70 and only $4 \%$ aged 49 years or younger according to the Surveillance, Epidemiology, and End Result (SEER) program. Atypical cases wherein the patient is less than 50 years old can occur 


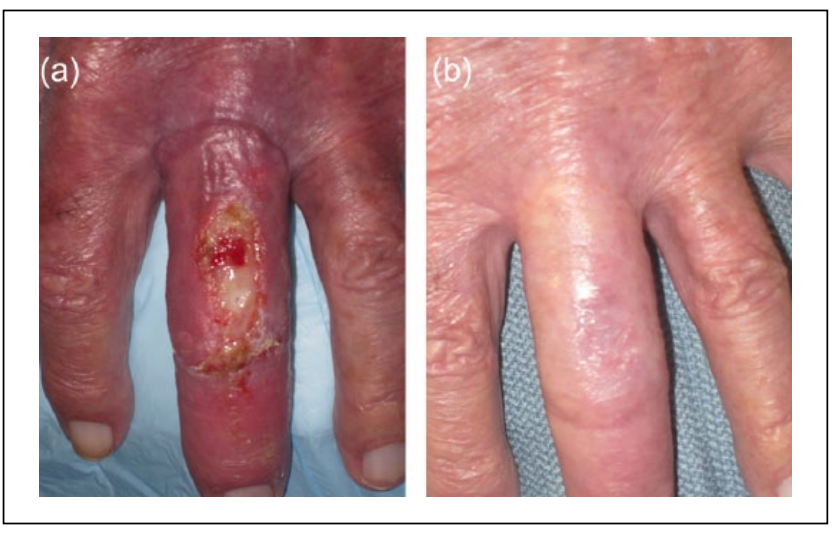

Figure 3. Follow-up at (a) 2 months demonstrating early wound healing with a small area of epidermolysis and (b) 6 months demonstrating final wound condition and excellent functional result.

in transplant patients. ${ }^{22}$ Furthermore, males appear to be at greater risk of MCC $(61.5 \%)$ compared with females $(38.5 \%),{ }^{2,18}$ and Caucasians account for $95 \%$ of the cases. ${ }^{2}$

Highly aggressive, MCC-specific mortality appears to be directly correlated to the microscopic spread of the disease, which follows the Halstedian model of spread with a stepwise spread to the regional lymph nodes prior to metastasizing hematogenously. ${ }^{24} \mathrm{~A}$ cohort study of over 5800 MCC patients outlines the relative survival rates of patients presenting with local disease, regional nodal disease, and distant metastatic disease. ${ }^{18}$ Patients presenting with local disease had $71 \%$ relative survival 3 years after MCC, whereas individuals with nodal and distant metastatic spread showed $48 \%$ and $20 \%$ relative survival rates, respectively, over the same time period. Initial tumor size also has been shown to have a positive predictive value on survival rates. Three years after diagnosis, individuals presenting with a $\leq 2-\mathrm{cm}$ lesion had a $74 \%$ survival rate, whereas those with a tumor sized 2.1 to $5.0 \mathrm{~cm}$ had a $62 \%$ survival rate. The predominant anatomical sites that present with MCC are areas with frequent sun exposure such as the skin of the face (27\%), skin of upper limb and shoulder $(22 \%)$, and skin of lower limb and hip $(15 \%) .^{2}$ In addition, incidence is increased in individuals of European descent, which may be related to the deficiency of protective melanocytes. With a high density of Merkel cells on the hand and frequent exposure of the hands to UV radiation, the likelihood of developing MCC on the hand is increased. Interestingly, a recent case report demonstrated the association of radiation exposure with MCC in a retired surgeon who frequently performed digestive tract radiography using the naked hand. ${ }^{4}$ The chronic exposure to radiation may have contributed to the development of MCC with squamous cell carcinoma in situ. Although rare, MCC has also been found infrequently on the penis, vulva, and in the parotid gland. . $^{5,10,14}$
Macroscopically, MCC presents most commonly as an asymptomatic nodular red/pink lesion $<2 \mathrm{~cm}$ in size, which demonstrates rapid growth of the tumor within weeks to months. ${ }^{12}$ Microscopically, it is characterized by small and round to oval cells disposed as either a discohesive population or as cohesive clusters of cells. ${ }^{16}$ The tumor cells contain nuclei exhibiting finely granular and dispersed chromatin without prominent nucleoli, scant cytoplasm, in addition to intercellular junctions. ${ }^{8,16,25}$ However, these cytomorphologic characteristics are not specific to MCC as they could present in other malignancies such as melanoma, small cell carcinoma, and lymphoma. Immunohistochemistry serves as a way to differentiate MCC from other similar carcinomas. Immunohistochemically, tumor cells demonstrating immunoreactivity against CK20 often allow for the positive identification of MCC. ${ }^{16}$

Cytogenetically, a recent report of MCC shows a complex karyotype with multiple chromosomal gains. A composite karyotype of $52, \mathrm{XY},+\mathrm{Y},+6,+6,+12,+19,+20$ was indicated. ${ }^{19}$ There is no cytogenetic similarity between primary MCC and metastatic MCC. Therefore, the extent of chromosomal aberrations may be related to the severity of the disease, as others report chromosomal translocations and deletions, and loss of heterozygosity of $3 \mathrm{p} 21$, to be present in MCC. ${ }^{29}$ Despite the lack of similarities, structural aberration in chromosome 1 is the most common cytogenetic finding in primary MCC. ${ }^{19}$

Surgical management of both primary site and SLN is the standard method of treatment for MCC. Current National Comprehensive Cancer Network (NCCN) guidelines recommend WLE of the primary site with 1 to $2 \mathrm{~cm}$ margins to the investing fascia of muscle when clinically feasible. For occurrences of MCC on the face or in other instances where tissue sparing is of high importance, histologic margin assessment may be warranted using Mohs technique. A retrospective analysis of 5823 cases indicates that $27 \%$ of cases presented with nodal disease. ${ }^{18}$ However, the management of a positive SLN remains an area of controversy. Complete excision of the primary site while achieving negative margins $(1-2 \mathrm{~cm})$ trends toward an overall improvement in long-term survival. ${ }^{26,27}$ Therefore, the initial evaluation of a presenting patient with suspected MCC includes the management of the primary tumor with wide local incision (1-2 $\mathrm{cm}$ margin) in addition to the management of the draining nodal basin with SLNB. For those individuals presenting with a positive SLN, a multidisciplinary tumor board consultation followed by node dissection and/ or radiation to the therapy to the tumor basin should be performed. Although the SLNB is an important staging tool, the importance in overall survival remains unclear. The lack of certainty regarding the management of lymph nodes differs from current guidelines of many other malignancies where lymph node dissection is indicated. The NCCN does recommend lymph node dissection for 
clinically evident lymphadenopathy in the axilla or groin, which should be followed by radiation therapy. However, the management of a microscopically positive node in which small metastatic foci of cells are present without extracapsular extension, the lack of clinically palpable lymphadenopathy, and lack of abnormal imaging criteria (computed tomography [CT], magnetic resonance imaging [MRI], or PET/CT scan) are less clear, but currently recommended to undergo radiation. In the event that distant metastases are identified, the current NCCN guidelines suggest considering adjuvant chemotherapy with cisplatin or carboplatin with or without etoposide. The rarity of MCC and lack of comprehensive randomized control studies hampers the establishment of guidelines for optimal therapy of those individuals afflicted with distant metastasis of MCC. In cases of in-transit metastasis, which are tumor cells distinct from their primary lesion, located within the lymphatic drainage route between the primary tumor and distant metastases, radiotherapy is ineffective. In such an event, palliative amputation of the limb in question may be justified. $^{11}$

The surgical approach in this patient was to outline the visible and palpable lesion on the dorsal finger. A second incisional outline was then drawn 1 to $2 \mathrm{~cm}$ from the tumor outline. Subsequently, excision the outlined lesion was performed in a full-thickness fashion removing the skin and subcutaneous tissue; however, the epitenon remained, as there was no evidence of adherence to the underlying tendon. Because of its high rate of recurrence, postoperative radiotherapy to the primary site and draining nodal regions was performed. This is in agreement with the current guidelines outlined by the NCCN. A 45- to 60-Gy dose has been shown to be beneficial. ${ }^{7}$

Multiple factors must be considered when closing a tumor defect after wide local incision. In this case, considerations included age, comorbidities, medical history, and need for radiation therapy. An FTSG was deemed appropriate for the patient given the relatively short procedure time, ability to be performed under local anesthesia with sedation, and relative tolerance to the proposed skin graft. Often more complex options included a cross-arm flap, dorsal metacarpal artery pedicle flap, or free tissue transfers including a venous flap from the forearm. These alternative options add a level of complexity and increase operating time for the management of the tumor excision defect.

As MCC is a relatively rare tumor, there are no randomized controlled trials or studies upon which the radiation oncologist can definitively base decisions related to radiation dosing. Each case is unique with its own treatmentrelated considerations regarding local control, function, and cosmesis for both short term and long term. Radiation, by general consensus, is now accepted as part of the standard therapy for MCC following WLE and sentinel node evaluation or lymph node dissection. Numerous anecdotal studies have repeatedly demonstrated improved local control with adjuvant irradiation, though overall survival may be unaffected. Disease-specific survival, however, generally is shown to improve with the addition of radiation after WLE. Improved local control may not translate into overall survival as this entity tends to occur in the elderly or immunosuppressed populations that tend to have shortened survivals as a group in any circumstance. Local control is generally beneficial as uncontrolled local tumor results in painful, bleeding, infected, unsightly tumor sites with functional compromise all of which are very distressing to patients and their caretakers.

The typical doses for radiation are extrapolated from those used to treat pulmonary small cell carcinoma with techniques similar to squamous cell carcinoma of the skin. Anecdotal reports suggest dose response curves similar to other epithelial tumors in the range of 45 to $50 \mathrm{~Gy}$ at standard fractionation sizes (1.8-2.0 Gy per fraction). Larger fraction sizes are sometimes used for expedience in areas of low functional or cosmetic consequence, but the radiobiologic equivalent (RBE) should equate approximately 50 Gy at 2 Gy doses per fraction. Treatment given to functionally important tissues, as in our case over extensor tendons and digits, necessarily requires standard fraction sizes, immobilization devices, carefully selected electron energies, and the use of tissue equivalent bolus material to maximize dose distribution and minimize dose to deeper tissues not at significant risk. This process, though time-consuming, will maximize local control, functional outcomes, and cosmetic results, and minimize long-term fibrosis as well as short-term skin reaction.

For this particular patient, nearly constant self-administered physical therapy in the form of aggressive range of motion and stretching was recommended and faithfully followed both during and after radiation delivery. We suggested that he continue this on a permanent basis, but through 18 months at a minimum. We selected $6 \mathrm{MeV}$ electrons and $0.5 \mathrm{~cm}$ bolus to the primary site over the middle finger and a large area of the dorsal hand midsection after acquiring a treatment planning CT scan in the department with his hand in a specially fabricated fixation device in the treatment position. In addition, at the axillary portion of the field, we used a posterior axillary boost (PAB) to bring the midplane axillary dose up to 46 Gy. Alternatively, anteroposterior-posterolateral (AP/PA) fields could have been used to achieve an equivalent effective dose. Standard fraction sizes were used to minimize the risk of upper extremity edema in the long term as well as to minimize the local axillary skin reaction, which can be quite severe when larger fraction sizes are used. During the treatment course, an epitrochlear node was palpated and spontaneously regressed with observation alone.

Although it is rare, MCC is increasing in incidence over the last several decades. As such, practitioners involved in hand and dermatologic surgery should be aware of the 
condition and its appropriate therapies. Optimal treatment requires multidisciplinary management including a wide field of specialists such as dermatopathologist, radiation oncologist, and highly knowledgeable surgeons. Moreover, it is important to be aware that $\mathrm{MCC}$ has a high rate of recurrence with a reported $4 \%$ and $6 \%$ incidence of recurrence locally and regionally, respectively, and a worrisome $8 \%$ incidence of recurrent distant metastasis. ${ }^{7}$ Taking this into consideration, the NCCN recommends a thorough physical exam including a regional lymph node exam every 3 to 6 months for 2 years, followed by every 6 to 12 months thereafter. In our case, multidisciplinary teamwork allowed treatment with a combination of surgical excision and adjuvant radiation leading to disease-free survival at 2.5-year follow-up.

\section{Author Contributions}

Donevan R. Westerveld analyzed and interpreted the data regarding the patient's condition and wrote the first draft of the manuscript and constructed the figures. David J. Hall performed editing of the manuscript in preparation for publication and was a significant contributor to the final draft. Winston T. Richards performed examination and administered treatment to the patient and was a major contributor in writing and editing the manuscript. All authors read and approved the final manuscript prior to submission.

\section{Ethical Approval}

This is a case report assessing surgical technique and care of a patient and therefore ethical approval was not necessary.

\section{Statement of Human and Animal Rights}

No experiments were undertaken on humans or animals for this study.

\section{Statement of Informed Consent}

Written informed consent was obtained from the patient for use of patient medical data and imaging for educational and research purposes. A copy of the written consent is available and has been provided for review by the Editor-in-Chief of this journal.

\section{Declaration of Conflicting Interests}

The author(s) declared no potential conflicts of interest with respect to the research, authorship, and/or publication of this article.

\section{Funding}

The author(s) received no financial support for the research, authorship, and/or publication of this article.

\section{References}

1. Agelli M, Clegg LX, Becker JC, Rollison DE. The etiology and epidemiology of Merkel cell carcinoma. Curr Probl Cancer. 2010;34(1):14-37.
2. Albores-Saavedra J, Batich K, Chable-Montero F, Sagy N, Schwartz AM, Henson DE. Merkel cell carcinoma demographics, morphology, and survival based on 3870 cases: a population based study. J Cutan Pathol. 2010;37(1):20-27.

3. Allen PJ, Bowne WB, Jaques DP, Brennan MF, Busam K, Coit DG. Merkel cell carcinoma: prognosis and treatment of patients from a single institution. $J$ Clin Oncol. 2005;23(10):2300-2309.

4. Ansai S, Noro S, Ogita A, Fukumoto H, Katano H, Kawana S. Case of Merkel cell carcinoma with squamous cell carcinoma possibly arising in chronic radiodermatitis of the hand. J Dermatol. 2015;42:207-209.

5. Azevedo Cavalcanti Reis F, Quirino R, Monnerat Lott F, Ornellas AA, Arcuri R. [Merkel cell carcinoma of penis]. Prog Urol. 2004;14(4):558-560.

6. Bobos M, Hytiroglou P, Kostopoulos I, Karkavelas G, Papadimitriou CS. Immunohistochemical distinction between Merkel cell carcinoma and small cell carcinoma of the lung. Am J Dermatopathol. 2006;28(2):99-104.

7. Boyer JD, Zitelli JA, Brodland DG, D'Angelo G. Local control of primary Merkel cell carcinoma: review of 45 cases treated with Mohs micrographic surgery with and without adjuvant radiation. J Am Acad Dermatol. 2002;47(6):885-892.

8. Domagala W, Lubinski J, Lasota J, Giryn I, Weber K, Osborn M. Neuroendocrine (Merkel-cell) carcinoma of the skin. Cytology, intermediate filament typing and ultrastructure of tumor cells in fine needle aspirates. Acta Cytol. 1987;31(3):267-275.

9. Feng H, Shuda M, Chang Y, Moore PS. Clonal integration of a polyomavirus in human Merkel cell carcinoma. Science. 2008;319(5866):1096-1100.

10. Ghaderi M, Coury J, Oxenberg J, Spector H. Primary Merkel cell carcinoma of the parotid gland. Ear Nose Throat J. 2010;89(7):E24-E27.

11. Gunaratne DA, Howle JR, Venesse MJ. Merkel cell carcinoma: a case of palliative upper limb amputation in a patient with refractory in-transit metastases. Australas J Dermatol. 2016;57(2):E53-E56.

12. Heath M, Jaimes N, Lemos B, et al. Clinical characteristics of Merkel cell carcinoma at diagnosis in 195 patients: the AEIOU features. J Am Acad Dermatol. 2008;58(3):375-381.

13. Hodgson NC. Merkel cell carcinoma: changing incidence trends. J Surg Oncol. 2005;89(1):1-4.

14. Iavazzo C, Terzi M, Arapantoni-Dadioti P, Dertimas V, Vorgias G. Vulvar Merkel carcinoma: a case report. Case Rep Med. 2011;2011:546972.

15. Kanitakis J, Euvrard S, Chouvet B, Butnaru AC, Claudy A. Merkel cell carcinoma in organ-transplant recipients: report of two cases with unusual histological features and literature review. J Cutan Pathol. 2006;33(10):686-694.

16. Knoepp SM, Hookim K, Placido J, Fields KL, Roh MH. The application of immunocytochemistry to cytologic direct smears of metastatic Merkel cell carcinoma. Diagn Cytopathol. 2011;41(8):729-733.

17. Kouzmina M, Leikola J, Böhling T, Koljonen V. Positive sentinel lymph node biopsy predicts local metastases during the course of disease in Merkel cell carcinoma. J Plast Surg Hand Surg. 2013;47(2):139-143. 
18. Lemos BD, Storer BE, Iyer JG, et al. Pathologic nodal evaluation improves prognostic accuracy in Merkel cell carcinoma: analysis of 5823 cases as the basis of the first consensus staging system. J Am Acad Dermatol. 2010;63(5): 751-761.

19. Manor E, Bellaiche E, Bodner L. Cytogenetic findings of a primary Merkel cell carcinoma. Cancer Genet Cytogenet. 2006;169(1):78-80.

20. Morrison KM, Miesegaes GR, Lumpkin EA, Maricich SM. Mammalian Merkel cells are descended from the epidermal lineage. Dev Biol. 2009;336(1):76-83.

21. Ortin-Perez J, van Rijk MC, Valdes-Olmos RA, et al. Lymphatic mapping and sentinel node biopsy in Merkel's cell carcinoma. Eur J Surg Oncol. 2007;33(1):119-122.

22. Penn I, First MR. Merkel's cell carcinoma in organ recipients: report of 41 cases. Transplantation. 1999;68(11): 1717-1721.

23. Prieto Munoz I, Pardo Masferrer J, Olivera Vegas J, Medina Montalvo MS, Jover Díaz R, Pérez Casas AM. Merkel cell carcinoma from 2008 to 2012: reaching a new level of understanding. Cancer Treat Rev. 2013;39(5):421-429.

24. Senchenkov A, Barnes SA, Moran SL. Predictors of survival and recurrence in the surgical treatment of Merkel cell carcinoma of the extremities. J Surg Oncol. 2007;95(3):229-234.

25. Sidhu GS, Feiner H, Flotte TJ, et al. Merkel cell neoplasms. Histology, electron microscopy, biology, and histogenesis. Am J Dermatopathol. 1980;2(2):101-119.

26. Tai P. A practical update of surgical management of Merkel cell carcinoma of the skin. ISRN Surg. 2013;2013:850797.

27. Tai PT, Yu E, Tonita J, Gilchrist J. Merkel cell carcinoma of the skin. J Cutan Med Surg. 2000;4(4):186-195.

28. Toker C. Trabecular carcinoma of the skin. Arch Dermatol. 1972;105(1):107-110.

29. Van Gele M, Leonard JH, Van Roy N, et al. Combined karyotyping, CGH and M-FISH analysis allows detailed characterization of unidentified chromosomal rearrangements in Merkel cell carcinoma. Int J Cancer. 2002;101(2): 137-145. 